\title{
A 2-Year Observational Study in Patients Suffering from Idiopathic Pulmonary Fibrosis and Treated with Pirfenidone: A French Ancillary Study of PASSPORT.
}

Jouneau $\mathrm{S}^{1}$, Gamez $A S^{2}$, Traclet $\mathrm{J}^{3}$, Nunes $\mathrm{H}^{4}$, Marchand-Adam $\mathrm{S}^{5}$, Kessler $\mathrm{R}^{6}$, Israël-Biet $\mathrm{D}^{7}$, Borie $\mathrm{R}^{8}$, Strombom $I^{9}$, Scalori $A^{10}$, Crestani $B^{8}$, Valeyre $D^{4}$, Cottin $\mathrm{V}^{3}$

1

Respiratory Diseases Department, Pontchaillou Hospital, IRSET UMR 1085, Rennes 1 University, Rennes, France, stephane.jouneau@chu-rennes.fr.

Pulmonology, Montpellier University Hospital, Montpellier, France.

Pulmonology Department, National Coordinating Reference Center for Rare

Pulmonary Diseases, Louis Pradel Hospital, University Claude Bernard Lyon 1, Lyon, France.

Pulmonology Department, Constitutive Site of the Reference Center for Rare Lung Diseases, Avicenne Hospital, Paris University 13, Bobigny, France.

Pulmonology Department, Tours Regional University Hospital, Tours, France.

Pulmonology, Strasbourg University Hospital, Strasbourg, France.

Pulmonology Department, Georges Pompidou European Hospital, AP-HP, Paris Descartes University, Paris, France.

Pulmonology Department, Bichat Hospital, Paris, France.

Genentech, San Francisco, California, USA.

Roche, Welwyn Garden City, United Kingdom. 


\section{Abstract}

Background: The European observational, prospective PASSPORT study evaluated the longterm safety of pirfenidone under real-world conditions in idiopathic pulmonary fibrosis (IPF), over up to 2 years following its initiation. Objectives: The FAS (French Ancillary Study) assessed the clinical outcomes of IPF patients participating in PASSPORT $(n=192)$. Methods: Efficacy data were collected retrospectively and prospectively. The primary efficacy endpoints were: change in percent predicted forced vital capacity (FVC) and change in the distance travelled during the 6-min walk test (6MWD). Results: The mean baseline FVC was $71.7 \%$ of predicted value. The mean absolute change in the percentage of predicted FVC was $-2.4 \%$ and $-3.8 \%$ at months 12 and 24. The mean change in 6MWD was 8.6 and $3.1 \mathrm{~m}$ at months 12 and 24 , with a range of 23.4-51.7 m. Acute IPF exacerbation and pulmonary hypertension occurred in 20.0 and $8.4 \%$ of patients, respectively. The most common reasons for prematurely discontinuing PASSPORT were adverse drug reactions (ADRs) related to pirfenidone (31.3\%), death (11.5\%), and disease progression (10.9\%). The median progression-free survival was 18.4 months (95\% Cl 12.9, not estimable). The median exposure was 16.3 months (0.5-28.5). The most frequently reported ADRs leading to pirfenidone discontinuation were decreased weight (4.2\%), rash (4.2\%), and photosensitivity reactions (3.1\%). Conclusions: The efficacy data of FAS are consistent with the efficacy results of published phase III clinical trials in IPF. Approximately one third of IPF patients treated with pirfenidone in real-life settings were still under treatment 2 years after initiation. Safety data are consistent with the known safety profile of pirfenidone.

\section{Introduction}

Idiopathic pulmonary fibrosis (IPF) is one of the most common forms of interstitial lung disease. This chronic, irreversible, debilitating disease of unknown etiology has a poor 
prognosis. It is associated with significant morbidity and mortality with a median survival estimated at approximately 3 years from diagnosis [1, 2]. Epidemiologic studies indicate that the current incidence estimated between 0.5 and 27.9 per 100,000 and prevalence $(0.22$ and 8.8 per 100,000$)$ of IPF is increasing [3-7]. IPF commonly occurs in patients aged 60 years or older. The disease onset is often slow, symptoms gradually worsen over time, and the clinical course and progression of IPF are highly variable [2]. Consequently, treatment strategies must be determined individually for each patient according to their medical history and specific comorbidities.

Until recently, treatment options for IPF were very limited, including mainly palliative care and lung transplant [8]. The advent of two oral treatment options, pirfenidone, an antifibrotic and anti-inflammatory agent, and nintedanib, a tyrosine kinase inhibitor, has transformed the treatment landscape for this disease [8]. Both drugs were approved for IPF in the USA and in Europe. They are recommended in the guidelines of the American Thoracic Society, the European Respiratory Society, the Japanese Respiratory Society, and the Latin American Thoracic Association (ATS/ERS/JRS/ALAT) updated in 2015 [9].

Evidence of the efficacy of pirfenidone has been demonstrated by four randomized, double-blind, placebo-controlled phase III trials collectively involving 1,067 patients in the two CAPACITY trials [10], a program of 2 multinational, randomized, double-blind, placebocontrolled, phase III trials involving 779 IPF patients [10], the ASCEND trial [11], and 1 phase III trial conducted in Japan [12]. These trials showed that pirfenidone significantly reduced the rate of decline of forced vital capacity (FVC) [10-12], as well as the risk of respiratory-related hospitalization [13]. In a meta-analysis [8] of the three largest trials, pirfenidone was further shown to significantly decrease mortality.

The PASSPORT registry, a European postmarketing safety study, was initiated at the request of the European Medicines Agency (EMA), in order to evaluate the safety profile of patients with IPF with long-term pirfenidone use, and to monitor for potentially new or unknown 
risks throughout a 2-year follow-up period [14]. The FAS (French Ancillary Study), an observational and ancillary study to the PASSPORT registry, was implemented in order to improve understanding of the clinical outcomes of IPF patients treated with pirfenidone under real-world conditions.

\section{Materials and Methods}

The PASSPORT registry (NCT02699879) was an observational, multicenter, prospective, postauthorization safety study of IPF patients treated with pirfenidone, monitored for up to 2 years. The study was conducted in 10 EU countries, including France. The present FAS was a non-interventional, observational, and ancillary study to the PASSPORT registry. It was implemented with the aim of collecting efficacy data as requested by the French authorities and was only conducted at French sites that were already participating in the PASSPORT registry.

For enrolment in the PASSPORT registry, patients had to have been newly prescribed pirfenidone therapy with initiation $\leq 30$ days prior to study enrolment. The decision to prescribe pirfenidone was made prior to enrolment. Patients were excluded if they were receiving an investigational agent for any indication or pirfenidone $>30$ days prior to enrolment or had a contraindication for pirfenidone use. For enrolment in the FAS, patients had to have been enrolled in the PASSPORT registry, to have received at least one dose of pirfenidone while participating in the PASSPORT registry, and to provide additional written informed consent for participation in this study, unless they were deceased or lost to follow-up.

Efficacy data were collected retrospectively and prospectively while patients completed up to 2 years of follow-up in PASSPORT. Data were collected at baseline, every 3 months $( \pm 4$ weeks) for up to 2 years and at early discontinuation visits, and at the end of the study. All data were collected using an electronic case report form, to supplement the data already being collected in PASSPORT. 
The primary efficacy endpoints were the change in percent predicted FVC and the change in the distance travelled during the 6-min walk test (6MWD) over the total follow-up. Secondary efficacy endpoints included the New York Heart Association (NYHA) functional class, Gender Age Physiology (GAP) score, time to IPF complications with specific emphasis on acute exacerbation and precapillary pulmonary hypertension, number and duration of IPFrelated hospitalizations, and progression-free survival (PFS). PFS was defined as the time from pirfenidone initiation until first occurrence of one of the following events: an absolute decline in percent predicted FVC $\geq 10 \%$, an absolute decline in $6 \mathrm{MWD} \geq 50 \mathrm{~m}$, or death from any cause. No imputation was made for missing data. Ad hoc exploratory efficacy endpoints that were analyzed included overall survival, and changes in FVC, forced expiratory volume in $1 \mathrm{~s}\left(\mathrm{FEV} \mathrm{V}_{1}\right)$, and diffusing capacity for carbon monoxide (DLCO) over the total treatment time (up to 2 years).

The modalities used to confirm the IPF diagnosis by the Multidisciplinary Diagnosis Discussion (certain, probable, possible, or unclassifiable idiopathic diffuse interstitial lung disease) as well as adverse drug reactions (ADRs) were two additional endpoints. All statistical analyses are descriptive in nature. No formal hypothesis testing was performed.

All patients enrolled in the FAS study were provided with a patient information sheet and had signed the consent form to have pirfenidone efficacy data collected in addition to their safety data. The Consulting Committee on the Handling of Information related to Health Research (French acronym: CCTIRS) approved the study on January 15, 2015, and the National Commission for Information Technology and Freedoms (French acronym: CNIL) approved it on May 12, 2015.

This study was conducted in accordance with good clinical practices as defined by the International Conference on Harmonization (ICH-GCP), the Helsinki Declaration, and in compliance with the Code of Public Health, Title II of the First Book on biomedical research. The PASSPORT study was registered on clinitrials.gov (NCT02699879). 


\section{Results}

A total of 214 patients were included in the PASSPORT registry across 22 sites in France. Among those, 192 patients were enrolled in this study, 63 (32.8\%) of whom completed the PASSPORT registry, while 22 patients did not sign the informed consent for the FAS study. The most common primary reasons for early discontinuation from the PASSPORT registry were ADRs related to pirfenidone (31.3\%), death (11.5\%), and disease progression (10.9\%; online suppl. Fig. S1; for all online suppl. material, see www.karger.com/doi/10.1159/000496735). The attrition rate and the reasons for discontinuation in the FAS study were similar to those of the PASSPORT study.

The mean age was 70.6 years, with $76.0 \%$ of patients aged 65 years or older. The majority of patients were male (84.4\%), 68.6\% were former smokers, and $3.1 \%$ were current smokers (Table 1). The median duration of IPF since diagnosis was 0.6 years, with a maximum of 12.2 years. A surgical lung biopsy was performed in $25.0 \%$ of patients. The diagnosis was confirmed in a multidisciplinary meeting in $81.7 \%$ of cases, and the diagnosis of IPF was determined as being certain, probable, or possible in $73.1,17.9$, and $7.1 \%$ of those, respectively.

At baseline, the majority of patients (54.8\%) had dyspnea staged NYHA II or III; however, the stage of dyspnea was reported as unknown for $35.8 \%$ of patients. One fifth of patients (19.8\%) were using supplementary oxygen. A history of sleep apnea was reported in $10.5 \%$ of patients, combined pulmonary fibrosis and emphysema syndrome in $10.1 \%$, acute IPF exacerbation in 6.9\%, precapillary pulmonary hypertension in $6.3 \%$, and lung cancer in $1 \%$. The mean baseline FVC was $71.7 \pm 16.3 \%$ of predicted value and $<50 \%$ for $9.9 \%$ of patients, $>80 \%$ for $30.9 \%$, and between 60 and $80 \%$ for $42.5 \%$ of patients (Table 1 ). Slightly more than half of the patients (52.1\%) had a baseline 6MWD, and the mean distance walked in 6 min was 412.9 m (range 105-702). 


\section{Efficacy}

The number of patients with postbaseline assessments of efficacy endpoints decreased over time. Only values in patients who completed up to 2 years of treatment with pirfenidone had to be collected. On average, absolute change from baseline in the percentage of predicted FVC varied from 0.18 to $-6.0 \%$. The mean absolute change from baseline in the percentage of predicted FVC was -2.4 at month 12 and -3.8 at month 24 (Fig. 1).

The proportion of enrolled patients who had died or showed an absolute decline $\geq 10 \%$ was $6.3 \%$ (12/192 patients) at months 3 and 6 , and then increased to between $14.6 \%(28 / 192$ patients) and $16.1 \%$ (31/192 patients) at months 9 and 12; it remained globally stable in the second year, from 15.6\% (30/192) at month 15 to 15.1\% (29/192) at month 24 (Fig. 1).

The mean change in 6MWD was $8.6 \mathrm{~m}$ at month 12 and $3.1 \mathrm{~m}$ at month 24 (Fig. 1), with a range of $23.4 \mathrm{~m}$ to $-51.7 \mathrm{~m}$. The proportion of patients who had died or had a decline of $\geq 50$ $\mathrm{m}$ in the 6MWD was $9.4 \%$ at month 12 and $12.5 \%$ at month 24 (Fig. 1).

At each time point from month 3 to month 24, the majority of patients had dyspnea staged NYHA II (from 50.0 to $63.0 \%$ ) or III (from 22.2 to $31.5 \%$ ). At baseline, $66.7 \%$ of patients were GAP stage II, $20.6 \%$ were stage III, and $12.7 \%$ were stage I.

During the follow-up, acute IPF exacerbation and precapillary pulmonary hypertension were reported in 20.0 and $8.4 \%$ of patients, respectively (Table 2). A total of 58 IPF-related hospitalizations were reported in 41 patients (21.4\%) during the study (Table 3). The majority of hospitalizations (56.9\%) lasted between 1 and 7 days, and 37.3\% lasted between 8 and 14 days. Hospitalizations related to respiratory disorders were the most frequent and occurred in 36 patients (18.8\%).

In total, $40.6 \%$ of patients had a disease progression, including $34.9 \%$ whose lung function declined and $5.7 \%$ who died without prior lung function decline. The median PFS was 18.4 months (95\% Cl 12.9, not estimable), and PFS rates at months 6, 12, and 24 were 85, 67, and $46 \%$, respectively (Fig. 2). 
The mean change from baseline in FVC, $\mathrm{FEV}_{1}$, and DLCO as well as overall survival were exploratory endpoints. The mean change from baseline in FVC was $-91 \pm 523 \mathrm{~mL}$ at month $12(n=79)$, and $-142 \pm 463 \mathrm{~mL}$ at month $24(n=36)$. The mean change from baseline in $\mathrm{FEV}_{1}$ was $-143 \pm 380 \mathrm{~mL}$ at month $12(n=79)$ and $-94 \pm 365$ at month $24(n=35)$. The mean change from baseline in DLCO was $-0.28 \pm 1.173 \mathrm{mmoL} / \mathrm{min} / \mathrm{kPa}$ at month $12(n=54)$ and $0.36 \pm 0.800 \mathrm{mmoL} / \mathrm{min} / \mathrm{kPa}$ at month $24(n=23)$.

A total of 22 patients (11.5\%) died during the study. All were men, with a mean age at baseline of 71.2 years (min. 44, max. 90); for 14/22 (64\%) of these patients, death occurred following disease progression. The estimated probabilities of survival by Kaplan-Meier at months 12 and 24 were 92 and 83\%, respectively (online suppl. Fig. S2).

\section{Safety}

The median duration of exposure to pirfenidone was 16.3 months (0.5-28.5), with a maximum exposure of 28.5 months (2.37 years). Approximately one third $(32.3 \%)$ of patients had a dose reduction during the study, and $17.7 \%$ had a dose interruption (online suppl. Table S1). The median time of participation for the 129 patients who discontinued the study was 9.3 months (1-30). In this population $25.0 \%$ of patients stayed in the study between 12 and $\leq 24$ months, $17.7 \%$ of patients stayed between 6 and $\leq 12$ months, $12.0 \%$ stayed between 3 and $\leq 6$ months, and $10.0 \%$ stayed $\leq 3$ months.

A total of 482 ADRs (events deemed by the investigator to be at least possibly related to pirfenidone) were reported in 155 patients (80.7\%); 461 were non-serious ADRs in 154 patients (80.2\%), and 19 were serious ADRs in 11 patients (5.7\%). The most frequent ADRs were decreased weight (29.7\% of patients), decreased appetite (18.8\%), nausea (15.6\%), and fatigue (15.6\%; Table 4). Serious ADRs were most frequently related to cutaneous disorders $(n=6$, $3.1 \%$ of patients) including nummular eczema, erythema, photosensitivity reactions, rash, general rash, and papular rash (Table 5). A total of 63 ADRs led to the discontinuation of pirfenidone in 61 patients (31.8\%). The most frequently reported ADRs leading to the 
discontinuation of pirfenidone were decreased weight (4.2\%), rash (4.2\%), and photosensitivity reactions (3.1\%). No patient experienced an ADR leading to a fatal outcome (Table 5).

\section{Discussion}

The FAS study provides information on the efficacy of pirfenidone in patients with IPF when used for up to 2 years in a real-life setting. FAS is an ancillary study of the PASSPORT registry, which was a European observational prospective postmarketing safety study. FAS was designed to collect efficacy data whose collection was unplanned in the PASSPORT registry. Some data were collected retrospectively because the study started while PASSPORT was still ongoing, and this in combination with the observational design of the study may explain the high rate of missing baseline values.

The patients included were slightly older than those included in CAPACITY [10] and ASCEND [11], and the proportions of both men and former smokers were slightly higher. A videothoracoscopic surgical lung biopsy was performed in $25 \%$ of patients. In contrast with CAPACITY $[10,15]$ and ASCEND [11], patients with a percentage of predicted FVC $<50 \%$ were included, and accounted for $10 \%$ of the FAS population. Missing values were not replaced in this study, which is another key difference between this observational study and phase III trials with pirfenidone. Consequently, the comparison between the observed outcomes must be regarded with caution.

At month 12, the mean absolute change in the percentage of predicted FVC was $-2.4 \%$, and $16.1 \%$ had an absolute decline of at least $10 \%$ in the percentage of predicted FVC or had died. The mean absolute change in the percentage of predicted FVC was lower than that observed in CAPACITY (-7.0\%), and for newly treated patients in the RECAP open-label extension study $(-5.9 \%)[15,16]$. However, the proportion of patients who had died or had a decline of at least $10 \%$ in the percentage of predicted FVC was comparable to $16.5 \%$ of 
patients as observed in the ASCEND study, 16.3\% as observed in RECAP for newly treated patients, and $16.8 \%$ as observed in CAPACITY [15]. Similar trends were observed at month 18 , with an absolute change in the percentage of predicted FVC of $-3.2 \%$ in FAS and $-8.5 \%$ in CAPACITY, and a proportion of patients who had a decline of at least $10 \%$ in the percentage of predicted FVC or had died of $16.7 \%$ in FAS and $21 \%$ in CAPACITY. In an analysis of ASCEND with no imputation of missing data [17], the proportion of patients who had a decline of at least $10 \%$ in the percentage of predicted FVC was $11.1 \%$ in the pirfenidone arm and $25.9 \%$ in the placebo arm. In comparison with phase III results, the proportion of patients was lower for those with no decline in the percentage of predicted FVC.

The change in the 6MWD at month 12 and month 18 differed from that reported in the phase III trials. At month 12, the proportion of patients who had a decline of at least $50 \mathrm{~m}$ in the 6MWD or had died was more than 2.5 times lower than those of the phase III trials: 9.4 versus 25.9\% in ASCEND, and $24.8 \%$ in a pooled analysis of ASCEND and CAPACITY [18]. At month 18 the proportion was $11.5 \%$ in the FAS, and 36 and 33\% in study 004 and study 006 , respectively.

A pooled analysis of the 3 pivotal studies of pirfenidone revealed a reduction of respiratory-related hospitalization rates in patients treated with pirfenidone versus placebo [13] of 7 versus 12\% during the first year of therapy. Patients included in this study, treated in reallife settings and followed for up to 2 years, had a respiratory-related hospitalization rate of $19 \%$. This higher rate in the FAS study may be explained by the inclusion of some patients with more advanced disease, possibly more comorbidities in this real-life setting than in randomized studies, and a longer follow-up, and is consistent with our previous study demonstrating high rates of hospitalizations for IPF in France [19]. Analyses taking these 2 points into account may aid data interpretation.

The discussion of PFS results is limited due to the fact that only hazard ratios were reported for the comparison between pirfenidone versus placebo in the CAPACITY and 
ASCEND publications, and in subsequent pooled analyses. Moreover, the definition of progression in CAPACITY differed from that of the FAS. At month $12,67 \%$ of the patients in FAS had neither died nor experienced progression, which seems to be slightly less than the rate estimated from the curves published in phase III pirfenidone trials. Due to the small number of patients and the high attrition rate predictive factors were not assessed.

The high rate of missing values for efficacy endpoints during the follow-up, as well as the lack of a comparative arm, preclude objective interpretation of efficacy results. In addition, comparison with the findings of phase III trials is limited by the differences existing between endpoints, statistical methods utilized, and the presentation of results. There were no differences between the characteristics of the patients included in the PASSPORT study and the subgroup of French patients included in the FAS study. Efficacy results and safety results were generally comparable. Notably, the guidelines on the drug therapy of IPF patients, whether French [20], from other European countries, or international [9], are very similar.

The safety data were consistent with the known safety profile of pirfenidone. The most frequent clinical ADRs were decreased appetite, gastrointestinal events (nausea, diarrhea), general disorders (fatigue, asthenia), and dermatological events (rash, photosensitivity). Despite an increased length of treatment exposure, the incidence of serious ADRs was lower than in the phase III trials, although adverse events leading to pirfenidone discontinuation were more frequent. However, slightly more patients in the FAS study discontinued treatment due to photosensitivity ( 2.1 vs. $0.5 \%$ ). The safety profile was similar to the known safety profile of pirfenidone $[21,22]$. No patient experienced an ADR with a fatal outcome.

This study has some limitations, including the retrospective collection of efficacy data as an ancillary study of a prospective, observational study, which caused some missing data. For example, only $52.1 \%$ of patients had 6MWD data at baseline, thus limiting the interpretation of results. The quality of the data was however better than in retrospective studies. Due to the study design, patients who did not tolerate the drug were excluded from the study when 
treatment was discontinued, which may have induced bias in the reporting of adverse events and possibly of survival. Conversely, as this study was part of a phase IV observational, prospective study evaluating the long-term safety of pirfenidone under real-world conditions and requested by regulatory authorities, particular attention was paid to exhaustive reporting of safety and tolerance, which may explain the relatively high frequency of adverse events in the study. The assessment of efficacy is limited by the design in the absence of a control group; however, such a real-life observational study provides reassurance since safety, tolerance, and outcome of efficacy data were consistent with that of randomized studies, and comparable with other observational studies [23-26]. The assessment of efficacy was performed in patients who remained on pirfenidone in the study, and attrition of the study population observed over the 2year follow-up may lead to overestimation of the stability of FVC and 6MWD.

In conclusion, efficacy data of the FAS are overall consistent with the efficacy results of published phase III clinical trials in IPF. Approximately one third of IPF patients treated with pirfenidone in real-life settings were still under treatment 2 years after initiation. FAS is one of the rare studies assessing efficacy in patients treated with pirfenidone in daily practice. The safety data were consistent with the known safety profile of pirfenidone.

\section{Acknowledgements}

The authors are grateful to the physicians, patients and their families who participated in this study. The authors would like to thank the study project team and Eltium for the support received in writing and editing this article.

\section{Statement of Ethics}




\section{Funding Sources}

The study was funded by Roche, France.

\section{Investigators}

Bergot Emmanuel, Investigator (Caen); Bonniaud Philippe, Investigator (Dijon); Borie Raphael, Investigator (Paris); Bourdin Arnaud, Investigator (Montpellier); Chanez Pascal, Investigator (Marseille); Cottin Vincent, Investigator (Lyon); Couturaud Francis, Investigator (Brest); Crestani Bruno, Co-investigator (Paris); Dromer Claire, Investigator (Bordeaux); Gagnadoux Frederic, Investigator (Vannes); Gamez Anne-Sophie, Co-investigator (Montpellier); Gondouin Anne, Investigator (Besançon); Israel-Biet Dominique, Investigator (Paris); Jouneau Stephane, Investigator-Co-ordinator (Rennes); Kessler Romain, Investigator (Strasbourg); Khouatra Chahera, Co-investigator (Lyon); Lebargy Francois, Investigator (Reims); MarchandAdam Sylvain, Investigator (Tours); Melloni Boris, Investigator (Limoges); Naccache Jean-Marc, Investigator (Paris); Nieves Ana, Co-investigator (Marseille); Nunes Hilario, Investigator (Bobigny); Prevot Grégoire, Investigator (Toulouse); Reynaud-Gaubert Martine, Investigator (Marseille); Tazi Abdellatif, Investigator (Paris); Traclet Julie, Co-investigator (Lyon); Urban Thierry, Co-investigator (Vannes); Valeyre Dominique, Co-investigator (Bobigny); Wallaert Benoit, Investigator (Lille).

\section{References}

1 Raghu G, Collard HR, Egan JJ, Martinez FJ, Behr J, Brown KK, et al.; ATS/ERS/JRS/ALAT Committee on Idiopathic Pulmonary Fibrosis. An official ATS/ERS/JRS/ALAT statement: idiopathic pulmonary fibrosis: evidence-based guidelines for diagnosis and management. Am J Respir Crit Care Med. 2011 Mar;183(6):788-824. 
2 Sharif R. Overview of idiopathic pulmonary fibrosis (IPF) and evidence-based guidelines. Am J Manag Care. 2017 Jul;23(11 Suppl):S176-82.

3 Kaunisto J, Salomaa ER, Hodgson U, Kaarteenaho R, Myllärniemi M. Idiopathic pulmonary fibrosis - a systematic review on methodology for the collection of epidemiological data. BMC Pulm Med. 2013 Aug;13:53

4 Hopkins RB, Burke N, Fell C, Dion G, Kolb M. Epidemiology and survival of idiopathic pulmonary fibrosis from national data in Canada. Eur Respir J. 2016 Jul;48(1):187-95.

5 Hutchinson J, Fogarty A, Hubbard R, McKeever T. Global incidence and mortality of idiopathic pulmonary fibrosis: a systematic review. Eur Respir J. 2015 Sep;46(3):795806.

6 Hutchinson JP, McKeever TM, Fogarty AW, Navaratnam V, Hubbard RB. Increasing global mortality from idiopathic pulmonary fibrosis in the twenty-first century. Ann Am Thorac Soc. 2014 Oct;11(8):1176-85.

7 Raghu G, Chen SY, Hou Q, Yeh WS, Collard HR. Incidence and prevalence of idiopathic pulmonary fibrosis in US adults 18-64 years old. Eur Respir J. 2016 Jul;48(1):179-86.

8 Nathan SD, Albera C, Bradford WZ, Costabel U, Glaspole I, Glassberg MK, et al. Effect of pirfenidone on mortality: pooled analyses and meta-analyses of clinical trials in idiopathic pulmonary fibrosis. Lancet Respir Med. 2017 Jan;5(1):33-41.

9 Raghu G, Rochwerg B, Zhang Y, Garcia CA, Azuma A, Behr J, et al.; American Thoracic Society; European Respiratory society; Japanese Respiratory Society; Latin American Thoracic Association. An official ATS/ERS/JRS/ALAT clinical practice guideline: treatment of idiopathic pulmonary fibrosis. An update of the 2011 clinical practice guideline. Am J Respir Crit Care Med. 2015 Jul;192(2):e3-19.

10 Noble PW, Albera C, Bradford WZ, Costabel U, Glassberg MK, Kardatzke D, et al.; CAPACITY Study Group. Pirfenidone in patients with idiopathic pulmonary fibrosis (CAPACITY): two randomised trials. Lancet. 2011 May;377(9779):1760-9. 
11 King TE Jr, Bradford WZ, Castro-Bernardini S, Fagan EA, Glaspole I, Glassberg MK, et al.; ASCEND Study Group. A phase 3 trial of pirfenidone in patients with idiopathic pulmonary fibrosis. N Engl J Med. 2014 May;370(22):2083-92.

12 Taniguchi H, Ebina M, Kondoh Y, Ogura T, Azuma A, Suga M, et al.; Pirfenidone Clinical Study Group in Japan. Pirfenidone in idiopathic pulmonary fibrosis. Eur Respir J. 2010 Apr;35(4):821-9.

13 Ley B, Swigris J, Day BM, Stauffer JL, Raimundo K, Chou W, et al. Pirfenidone Reduces Respiratory-related Hospitalizations in Idiopathic Pulmonary Fibrosis. Am J Respir Crit Care Med. 2017 Sep;196(6):756-61.

14 Cottin V, Koschel D, Günther A, Albera C, Azuma A, Sköld CM, et al. Long-term safety of pirfenidone: results of the prospective, observational PASSPORT study. ERJ Open Res. 2018 Oct;4(4):00084-2018.

15 Costabel U, Albera C, Bradford WZ, Hormel P, King TE Jr, Noble PW, et al. Analysis of lung function and survival in RECAP: an open-label extension study of pirfenidone in patients with idiopathic pulmonary fibrosis. Sarcoidosis Vasc Diffuse Lung Dis. 2014 Oct;31(3):198-205.

16 Costabel U, Albera C, Lancaster LH, Lin CY, Hormel P, Hulter HN, et al. An Open-Label Study of the Long-Term Safety of Pirfenidone in Patients with Idiopathic Pulmonary Fibrosis (RECAP). Respiration. 2017;94(5):408-15.

17 Lederer DJ, Bradford WZ, Fagan EA, Glaspole I, Glassberg MK, Glasscock KF, et al. Sensitivity Analyses of the Change in FVC in a Phase 3 Trial of Pirfenidone for Idiopathic Pulmonary Fibrosis. Chest. 2015 Jul;148(1):196-201.

18 Noble PW, Albera C, Bradford WZ, Costabel U, du Bois RM, Fagan EA, et al. Pirfenidone for idiopathic pulmonary fibrosis: analysis of pooled data from three multinational phase 3 trials. Eur Respir J. 2016 Jan;47(1):243-53. 
19 Cottin V, Schmidt A, Catella L, Porte F, Fernandez-Montoya C, Le Lay K, et al. Burden of Idiopathic Pulmonary Fibrosis Progression: A 5-Year Longitudinal Follow-Up Study. PLoS One. 2017 Jan;12(1):e0166462.

20 Cottin V, Crestani B, Cadranel J, Cordier JF, Marchand-Adam S, Prévot G, et al. French practical guidelines for the diagnosis and management of idiopathic pulmonary fibrosis 2017 update. Full-length version. Rev Mal Respir. 2017 Oct;34(8):900-68.

21 Summary of the Product Characteristics - Pirfenidone.

22 Lancaster LH, de Andrade JA, Zibrak JD, Padilla ML, Albera C, Nathan SD, et al. Pirfenidone safety and adverse event management in idiopathic pulmonary fibrosis. Eur Respir Rev. 2017 Dec;26(146):170057.

23 Tzouvelekis A, Karampitsakos T, Ntolios P, Tzilas V, Bouros E, Markozannes E, et al. Longitudinal "real-world" outcomes of pirfenidone in idiopathic pulmonary fibrosis in Greece. Front Med. 2017 Nov;4:213.

24 Galli JA, Pandya A, Vega-Olivo M, Dass C, Zhao H, Criner GJ. Pirfenidone and nintedanib for pulmonary fibrosis in clinical practice: tolerability and adverse drug reactions. Respirology. 2017 Aug;22(6):1171-8.

25 Harari S, Caminati A, Albera C, Vancheri C, Poletti V, Pesci A, et al. Efficacy of pirfenidone for idiopathic pulmonary fibrosis: an Italian real life study. Respir Med. 2015 Jul;109(7):904-13.

26 Oltmanns U, Kahn N, Palmowski K, Träger A, Wenz H, Heussel CP, et al. Pirfenidone in idiopathic pulmonary fibrosis: real-life experience from a German tertiary referral center for interstitial lung diseases. Respiration. 2014;88(3):199-207. 
Fig. 1. Change in percent predicted FVC (a) and 6MWD (b) over time (enrolled population). Baseline is the last assessment available up to and including the baseline visit day. Change from baseline was derived as follow-up minus the baseline value and, as such, a negative value indicates a decrease from baseline. Bars indicate standard errors of the mean change. Deaths from earlier time points are included in the categorical summaries, and percentages are based on the total number of patients enrolled.

Fig. 2. Kaplan-Meier plot of PFS (enrolled population). PFS was defined as the time from the first dose of pirfenidone to the first occurrence of: an absolute decline in percent predicted FVC $\geq 10 \%$ over the duration of follow-up, an absolute decline in $6 \mathrm{MWD} \geq 50 \mathrm{~m}$ over the duration of follow-up, or death from any cause. 
Table 1. Baseline characteristics (total enrolled population, $n=192$ )

Male gender

Age, years

Age group $\geq 65$ years

BMI $(n=191)$

Cigarette smoking history $(n=191)$

Present

Former

Never

Time since diagnosis, years Median (min.-max.)

IPF-related comorbidities

History of acute exacerbation $(n=189)$

Combined pulmonary fibrosis and emphysema syndrome $(n=188)$

Precapillary pulmonary hypertension $(n=190)$

Sleep apnea syndrome $(n=191)$

History of lung cancer $(n=192)$

NYHA dyspnea stage at baseline $(n=190)$

I

II

III

IV

Unknown

Distance walked in 6MWD ( $n=99), \mathrm{m}$

Supplemental oxygen at time of the 6MWD test $(n=99)$

DLCO (percent of predicted value; $n=137$ )

$\mathrm{FVC}^{\mathrm{a}}$ (percent of predicted value; $n=181$ )

$>80 \%$

$60-80 \%$

$50-60 \%$

$<50 \%$

Supplementary oxygen use

Concomitant pulmonary rehabilitation at baseline $(n=190)$

Yes
$162(84.4)$

$70.6 \pm 8.1$

$146(76.0)$

$27.5 \pm 4.0$

$6(3.1)$

$131(68.6)$

$54(28.3)$

$1.8 \pm 2.6$

$0.6(0.01-12.2)$

13 (6.9)

19 (10.1)

12 (6.3)

20 (10.5)

$2(1.0)$

15 (7.9)

67 (35.3)

37 (19.5)

3 (1.6)

68 (35.8)

$412.9 \pm 130.5$

10 (10.1)

$39.3 \pm 11.8$

$71.7 \pm 16.3$

56 (30.9)

77 (42.5)

30 (16.6)

18 (9.9)

38 (19.8)

$4(2.1)$

Data are presented as $n(\%)$, mean $\pm \mathrm{SD}$, or as indicated.

${ }^{a}$ If ethnicity was missing for the percent predicted FVC calculation, ethnicity was assumed to be Caucasian. 
Table 2. Summary of new or worsened IPF and IPF-related comorbidities (enrolled population, $n=190$ )

Acute exacerbation of IPF

$38(20.0)$

Combined pulmonary fibrosis and emphysema

$0(0.0)$

Precapillary pulmonary hypertension

$16(8.4)$

Sleep apnea syndrome

$1(0.5)$

Lung cancer

$9(4.7)$

Data are presented as $n(\%)$. IPF, idiopathic pulmonary fibrosis. The number of patients, $n$, with a non-missing response ("yes," "no," or "unknown") at the given visit is used as the denominator for the calculation of percentages. All observations, including those outside visit windows, were included. 
Table 3. Summary of IPF-related hospitalizations during the study (enrolled population, $n=192$ )

Total number of IPF-related hospitalizations

58

Length of each IPF-related hospitalization $(n=51)$, days

Mean (SD)

Median (min.-max.)

$8.2(8.5)$

1-7 days

$7.0(1-56)$

8-14 days

$29(56.9)$

$19(37.3)$

$>14$ days

$3(5.9)$

Number of IPF-related hospitalizations per patient

1

2

$29(15.1)$

$8(4.2)$

3 or more

4 (2.1)

Data are presented as $n(\%)$ or as indicated. IPF, idiopathic pulmonary fibrosis. 
Table 4. Most frequent ADRs (reported in $>2.5 \%$ of patients; enrolled population, $n=192$ )

\begin{tabular}{lrr}
\hline & Events, $n$ & \multicolumn{1}{l}{$\begin{array}{l}\text { Patients, } \\
n(\%)\end{array}$} \\
\hline Patients with any ADR & 482 & $155(80.7)$ \\
Decreased weight & 59 & $57(29.7)$ \\
Decreased appetite & 38 & $36(18.8)$ \\
Nausea & 31 & $30(15.6)$ \\
Fatigue & 30 & $30(15.6)$ \\
Asthenia & 29 & $28(14.6)$ \\
Diarrhea & 27 & $24(12.5)$ \\
Rash & 26 & $23(12.0)$ \\
Photosensitivity reaction & 21 & $21(10.9)$ \\
Erythema & 17 & $15(7.8)$ \\
Dizziness & 12 & $12(6.3)$ \\
Abdominal pain & 9 & $9(4.7)$ \\
Cough & 8 & $8(4.2)$ \\
Pruritus & 9 & $8(4.2)$ \\
Dyspepsia & 6 & $6(3.1)$ \\
Vertigo & 5 & $5(2.6)$ \\
Constipation & 5 & $5(2.6)$ \\
Headache & 5 & $5(2.6)$ \\
\hline \multicolumn{1}{c}{ ADR, adverse dug reaction. } & & \\
\hline
\end{tabular}




\begin{tabular}{lrr}
\hline & \multicolumn{2}{c}{$\begin{array}{r}\text { Events, } n \text { Patients, } \\
n(\%)\end{array}$} \\
\hline Patients with any serious ADR & 19 & $11(5.7)$ \\
Retinal artery occlusion & 1 & $1(0.5)$ \\
Edema peripheral & 1 & $1(0.5)$ \\
Respiratory tract infection viral & 1 & $1(0.5)$ \\
Alanine aminotransferase increased & 1 & $1(0.5)$ \\
Aspartate aminotransferase increased & 1 & $1(0.5)$ \\
Blood bilirubin increased & 1 & $1(0.5)$ \\
Gamma-glutamyltransferase increased & 1 & $1(0.5)$ \\
Basal cell carcinoma & 1 & $1(0.5)$ \\
Refractory cytopenia with multilineage & & \\
$\quad$ dysplasia & 1 & $1(0.5)$ \\
Dizziness & 1 & $1(0.5)$ \\
Neuralgia & 1 & $1(0.5)$ \\
Depression & 1 & $1(0.5)$ \\
Bronchospasm & 1 & $1(0.5)$ \\
Nummular eczema & 1 & $1(0.5)$ \\
Erythema & 1 & $1(0.5)$ \\
Photosensitivity reaction & 1 & $1(0.5)$ \\
Rash & 1 & $1(0.5)$ \\
Generalized rash & 1 & $1(0.5)$ \\
Papular rash & 1 & $1(0.5)$ \\
\hline ADR, adverse drug reaction. & & \\
\hline
\end{tabular}


res496735_F01_1.jpg
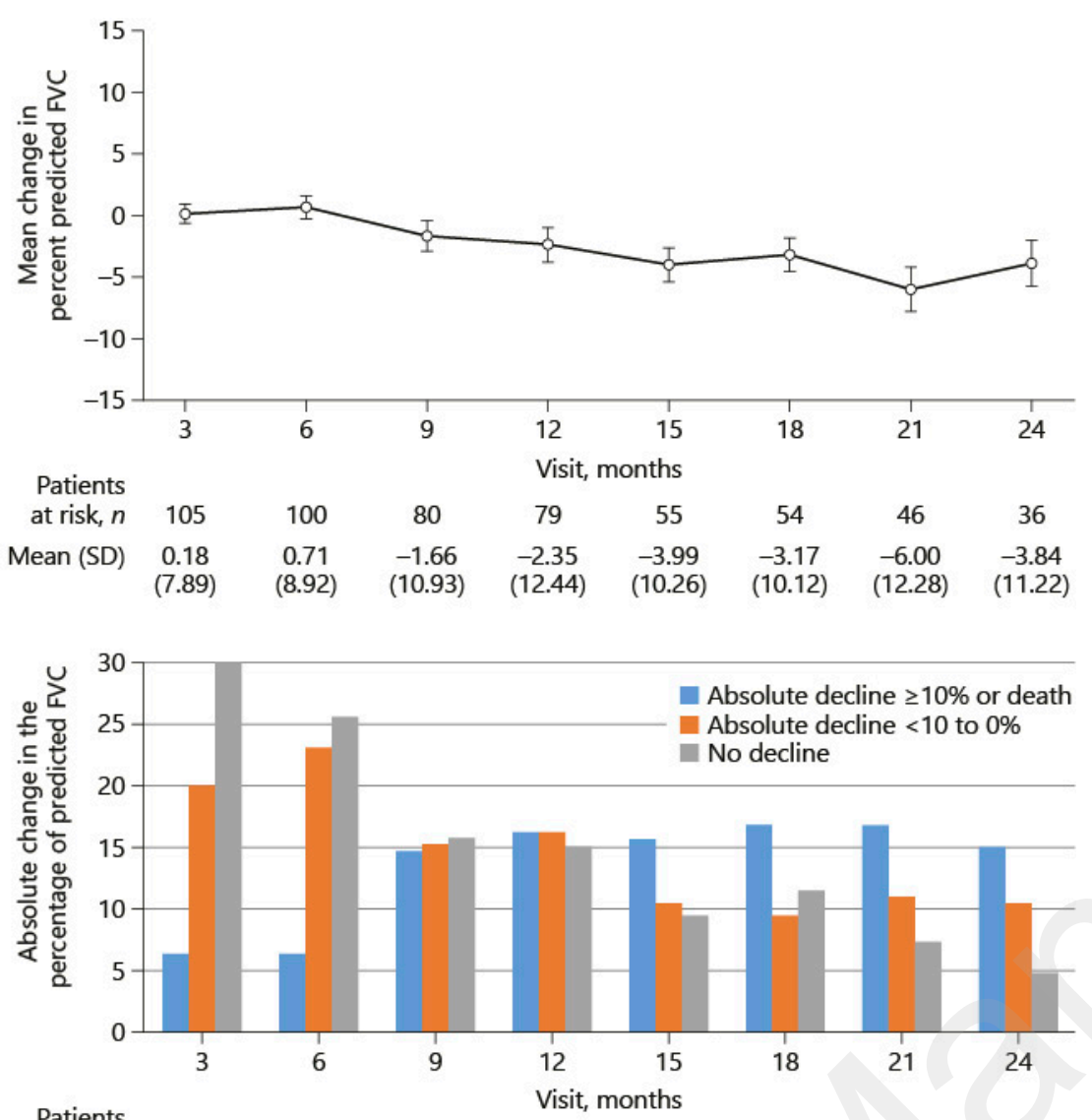

$\begin{array}{lllllllll}\text { a } \text { at risk, } n & 105 & 100 & 80 & 79 & 55 & 54 & 46 & 36\end{array}$

Fig. 1. Change in percent predicted FVC (a) and 6MWD (b) over time (enrolled population). Baseline is the last assessment available up to and including the baseline visit day. Change from baseline was derived as follow-up minus the baseline value and, as such, a negative value indicates a decrease from baseline. Bars indicate standard errors of the mean change. Deaths from earlier time points are included in the categorical summaries, and percentages are based on the total number of patients enrolled. 


\section{res496735_F01_2.jpg}
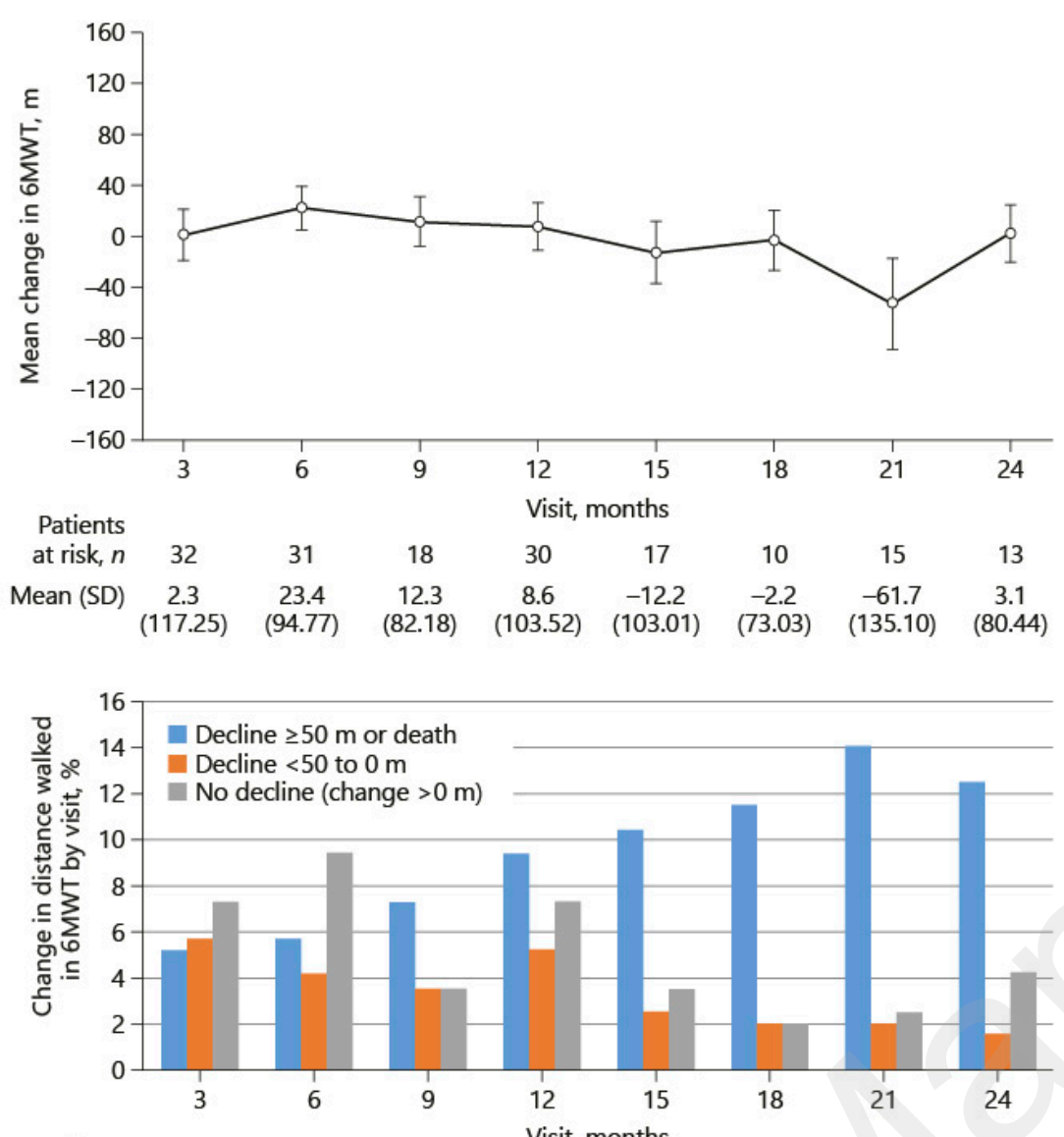

Patients

b at risk, $n$

32

31

isit, months

$10 \quad 15$

13 
res496735_F02.jpg

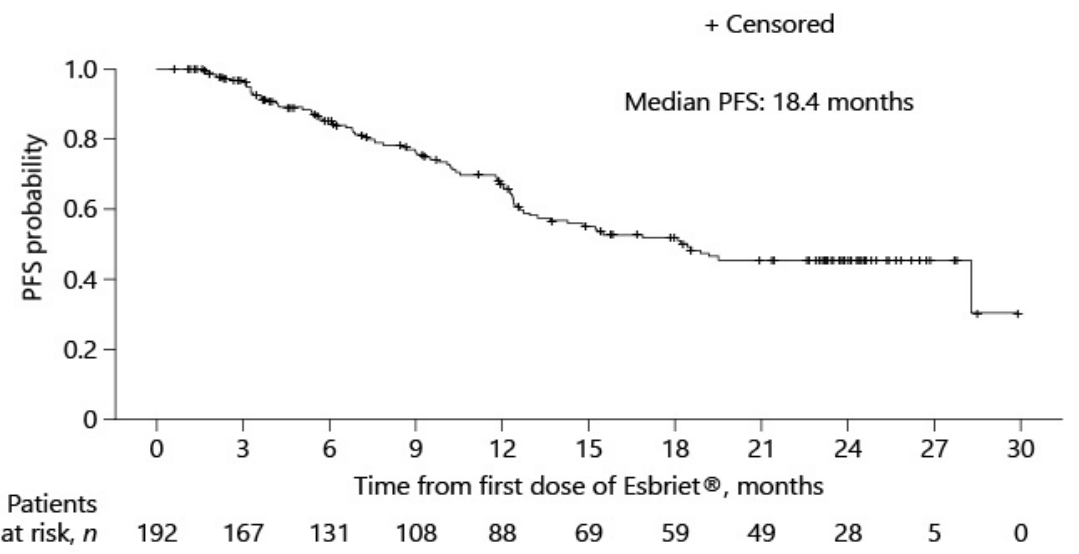

Fig. 2. Kaplan-Meier plot of PFS (enrolled population). PFS was defined as the time from the first dose of pirfenidone to the first occurrence of: an absolute decline in percent predicted FVC $\geq 10 \%$ over the duration of follow-up, an absolute decline in $6 \mathrm{MWD} \geq 50 \mathrm{~m}$ over the duration of follow-up, or death from any cause. 